\section{Modality effects in retrieval of information from short-term memory}

\author{
DAVID BURROWS* \\ University of Toronto, Toronto, Ontario, Canada
}

Retrieval from short-term memory was studied in a reaction time task. Ss decided whether or not a target item was present in a memorized list. When modality of presentation was manipulated, fastest reaction times resulted if the target item and all memory items were presented auditorily. All conditions involving pure visual presentation or mixed auditory-visual presentation resulted in reaction times which were longer than those for the pure auditory conditions but which did not differ from each other. This suggests that retrieval can be from either a precategorical auditory memory or a postcategorical memory which does not preserve the sensory features of the memorized material.

A crucial problem in understanding short-term memory (STM) concerns the format of memorized information. How can memorized material be characterized? If memory involves storage of information in some sense, what is it that is stored? While much research has been devoted to studying this problem through determinants of failure to remember (e.g., Conrad, 1964; Baddeley, 1966), little work has been done to investigate the format of remembered information in error-free situations. The present experiments attempt to study the format of remembered material under low error conditions using a technique developed by Sternberg (1966, 1969). $S$ is presented with a short series of items which he tries to memorize, followed by a single test or probe item. He must decide whether or not the probe item was one of the memory items, indicating his decision by pulling one of two levers as quickly as possible, Inferences about memory can be made by observing the mean reaction time (RT) of the response. Sternberg found that $\mathbf{R T}$ of the binary decision is a linear function of the number of items in memory. He inferred that $\mathbf{S}$ accomplishes the task by a scanning process of

*Now at the University of Minnesota, Center for Research in Human Learning. This paper is based on a PhD dissertation submitted to the University of Toronto. The author thanks his committee chairman, $B$. B. Murdock, Jr., and members of the committe, M. C. Smith and R. S. Lockhart, for assistance at all stages of this research. The research was supported by National Research Council of Canada Grant APA 146 to Dr. Murdock. The paper was written while the author was a postdoctoral fellow at the Center for Research in Human Learning, University of Minnesota. Preparation of this paper was supported in part by grants to the University of Minnesota, Center for Research in Human Mearning, from the National Science Foundation (GB-17590), the National Institute of Child Health and Human Development (HD-01136 and HD-00098), and the Graduate School of the University of Minnesota. comparing a representation of the probe item with internal representations of each memory item, each comparison requiring the same average amount of time. If the probe matches any memory item, a positive response is made. If no match is found, a negative response is made. The mean time per internal comparison is represented by the slope of $\overline{\mathrm{RT}}$ plotted against memory set size, where memory set size is defined as the number of items in memory.

The strategy of the present experiments is to make inferences about the format or storage properties of items held in STM by using a Sternberg procedure, manipulating the format of both memory and probe items and observing the effects on RT. The hypothesized comparison process should be executed in less time if probe and memory items are in a compatible format than if they are in an incompatible format. Given a group of probe items varying in format, that item which leads to the shortest $\overline{\mathrm{RT}}$ should be closest in format to the me mory items.

Changing the format of the probe item from one which is compatible with the memory items to one which is incompatible could affect the $\overline{R T}$ function in one of two ways: (1) If the probe item is recoded into a compatible format before scanning begins, the intercept of the $\overline{R T}$ function would be raised. (2) If scanning begins before the probe item is recoded, each comparison will take longer than if the formats were compatible and the slope of the $\overline{R T}$ function will be greater. It is, of course, possible that both intercept and slope will be raised if recoding is done before scanning begins but the recoded probe is still not completely compatible with the memory items. The principle manipulation of format used in the present experiments is to vary the modality of both memory items and test items to observe whether STM items are stored in an "auditory" format, a "visual" format, neither, or both.

Currently there are four major theories of memory format: auditory (e.g., Conrad, 1964; Sperling, 1963), visual (Stermberg, 1967), bimodal (Murdock, 1966, 1967, 1968, 1969), and "logogen" (Crowder \& Morton, 1969). Each theory is described briefly, along with the predictions it would make in a Sternberg procedure where modality of presentation is being manipulated. (1) Auditory: The main feature of auditory storage theories is that regardless of mode of input, all information is recoded into acoustic representations. Retrieval would involve comparison between probe items and a list of acoustic features or an auditory name which defines each memory item. Auditory probes should result in shorter $\overline{\mathrm{R} T s}$ than visual probes, and the modality in which the memory items are presented should have no effect. (2) Visual: Although there is little evidence that all memory items are stored in a visual format, Sternberg (1967) has maintained that with visual presentation, the comparison process involved in scanning is carried out along visual dimensions. Some other type of storage form might be converted into visual form when necessary. Sternberg has not commented on the case of auditory presentation; if the comparisons are still visual in nature, visual test items would lead to the shortest RT regardless of memory item mode of presentation. 1 (3) Bimodal: Murdock (1966, 1967, 1968, 1969) has argued that STM format preserves the features of input modality; there are separate stores for auditory and visual information. Such a model would predict that latency is shortest when memory and probe items are presented in the same modality. (4) Logogen: This model asserts that presented information is maintained in modality-specific stores for only a brief time before being fed into a "logogen" which categorizes the material and extracts semantic features. The visual sensory store preserves information for 1 sec or less while the auditory store can preserve information for several seconds. Since the logogen is compatible with neither auditory nor visual information, $\overline{\mathbf{R T}}$ should in general be equal for visual and auditory probes (assuming that conversion into logogen format requires equal time for the two modalities). However, retrieval of auditorily presented information from the auditory store could occur if the retention interval is not too long. An auditory probe might lead to shorter 
$\overline{\mathrm{RT}}$ than a visual probe if the memory items were presented auditorily. There would be no such comparable effect for visually presented memory items since the visual store is of too short duration.

These predicitons are for cases where all memory items are presented in one modality. If input is mixed so that on a given trial some memory items are presented auditorily and some visually, the predictions change. Models 1 and 2, which assert that input modality has no bearing on STM format, would predict no differences between mixed and "pure" (single modality) presentation conditions. The bimodal model should predict that the mixed condition is harder than the pure conditions; since memory items are stored in two formats, it is necessary to make some comparisons between probe and memory items across modality or to adopt some other change in strategy which would disrupt the normal scanning process. $\overline{\mathrm{RT}}$ should be longer in the mixed conditions than in the pure conditions. It is not clear what prediction the logogen model would make, but one strong possibility is that with mixed input, all retrieval is from the logogen store. The mixed condition would be equivalent to the conditions where memory input is pure visual or the input is auditory and the probe is visual.

\section{EXPERIMENT 1}

Experiment 1 tested the models of storage format in a Sternberg task where modality of both memory set items and probe was varied. On each trial, the presentation of memory set items was either all auditory, all visual, or mixed (some items presented auditorily, some visually). For each of the three types of memory set, probe items could be auditorily or visually presented.

Subjects. The Ss were 20 undergraduate students at the University of Toronto, both male and female. Each $S$ served in two sessions of $1 \mathrm{~h}$ each and was paid $\$ 2.00$ per session.

Apparatus. Stimulus lists were generated by computer. Visual stimuli were typed by the computer program, and the printouts were inserted into an IBM Selectric typewriter and displayed via a closed circuit TV camera focused on the carriage. Auditory stimuli were recorded on an Ampex FL-44 tape recorder. Each $S$ was seated in a soundproof chamber where he viewed the visual stimuli on a TV monitor and heard the auditory stimuli on Sharpe headphones. Control of presentation rate and synchronization of the auditory and visual stimuli was achieved by recording signals on the auditory tapes at the desired intervals. These signals were read and converted into electrical pulses by a Uher diapilot. The pulses activated the RETURN button on the typewriter, advancing the carriage. Whenever an auditory stimulus was being presented to $\mathrm{S}$, the TV monitor displayed a blank. Responses were made by pressing one of two telegraph keys. RTs were measured to the nearest millisecond.

Procedure. Each trial started with a row of asterisks on the TV monitor presented at the same time as the spoken word "start." The memory set followed at a rate of 3 items $/ 2$ sec. Following memory set presentation, there was a warning signal which consisted of a visual row of " $+s$ " and the simultaneously spoken word "probe." The "warning signal" lasted for $2 / 3$ sec and was followed by the probe item itself. $S$ was required to decide as quickly as possible whether the probe item was one of the memory set items and to indicate his decision by pressing one of the two keys. After a 4-sec interval, the next trial began. All stimulus items were single-digit numbers.

A complete replication consisted of a block of auditory memory set trials, a block of visual memory set trials, and a block of mixed memory set trials. A block of auditory memory set trials consisted of 16 trials in which all memory items were presented auditorily. Each possible combination of memory set size $(1,2,3$, or 4 items), probe type (pasitive or negative), and probe modality (auditory or visual) was represented once in the 16 trials. A block of visual memory items consisted of 16 trials in which all memory set items were presented visually with each combination of set size, probe type, and probe modality represented once.

For each trial in a block of mixed memory trials, some memory set items were presented auditorily and some visually. Since it is impossible to have a mixed memory set with a single memory item, the only set sizes used were 2,3 , or 4 items. In addition to varying the factors of probe type (positive or negative) and probe modality (auditory or visual), it was possible, on positive trials, to vary the modality in which the target item was originally presented. By adding the factor of modality of target items (if any), 3 by 2 by 2 by $2=24$ different types of mixed memory set trials resulted. There are several ways that mixed memory sets can be composed (a 3 -item set can be 2 auditory and 1 visual or 1 auditory and 2 visual; a 4-item set can be split $3-1,2-2$, or 1-3).
Across replications the frequency of each possible set composition was equal for each possible type of mixed set trial. On mixed set trials the order in which the auditory and visual items were presented was random, with the constraint that on positive trials the item in the serial position that would be tested was presented in the proper modality.

For all three types of memory set, serial position of the target item on positive trials was controlled across replications so that for each combination of trial type, each serial position was tested with equal frequency.

Each $S$ was tested on five replications per day. The first replication was considered practice and was discarded. The order in which the visual, auditory, and mixed blocks were presented within each replication was randomized.

Results

The error rates ranged from $6 \%$ to $15 \%$ over the various conditions with a mean of $10 \%$. In general, conditions leading to high error rates also led to long $\overline{R T s}$. In addition to errors, some Ss made occasional responses with extremely long latencies. All errors and responses with latencies of $2.5 \mathrm{sec}$ or longer were eliminated before the RT data were analyzed. The "long" responses constituted less than $2 \%$ of the total for any single $S$.

Figure 1 shows $\overline{\mathbf{R T}}$ to auditory probes as a function of memory set size for the pure auditory (AA) and pure visual (VA) memory set conditions. Data for positive and negative responses have been combined, since response type did not appear to affect $\overline{R T}$. The fitted straight. lines were determined by least-squares linear regression (Draper \& Smith, 1966). Linear regression accounted for $97.1 \%$ of the variance. The slope for the VA condition is almost $50 \%$ steeper than the slope for the AA condition. A t-test on the fitted functions indicated that the slopes are different, $t(4)=2.37$, $p<.05$, but that the intercepts of the two functions do not differ, $t(4)=0.33, n . s$.

Figure 2 shows $\overline{\mathrm{RT}}$ to visual probes, again comparing the conditions where memory set presentation was auditory (AV) and those where memory presentation was visual (VV). Again, data for positive and negative responses have been combined, since response type did not appear to have any effect. Linear regression accounted for $96.9 \%$ of the variance. Unlike the auditory probe conditions, modality of memory items seemed to have little effect when the probe was visual. Tests on the fitted lines indicated 
that neither the difference in slope, $t(4)=.21$, n.s., nor the difference in intercept, $t(4)=.85$, n.s., reached significance. The slopes for the AV and VV conditions are similar to the slope for the VA condition.

Figure 3 shows the RT functions for the conditions where the modality of me mory set items was mixed. Separate curves are shown for the auditory probe and visual probe conditions. The positive and negative functions have been combined, since probe type did not appear to have any effect. Linear regression accounted for $89 \%$ of the variance, reflecting the fact that the linear fits were not as good. The linear functions for the mixed mode cases have parameters similar to those for the VA, VV, and AV conditions. The AA condition appears to be unique while all other conditions fall into one cateogry. The slope values for all but the AA condition are also similar to the slope that Sternberg (1966) obtained for a VV task.

\section{Discussion}

The results of Experiment 1 suggest that in the AA condition, the mean time for each comparison is shorter than in any of the other conditions. There is one rate of scanning for the AA condition and a slower scanning rate for all of the other conditions. This difference in scanning rate as a function of input modality rules out any simple unitary store model. Such models imply that once an item is in STM its original mode of presentation is irrelevant. This is incompatible with the data for the auditory test item conditions which show that rate of retrieval is sensitive to presentation mode.

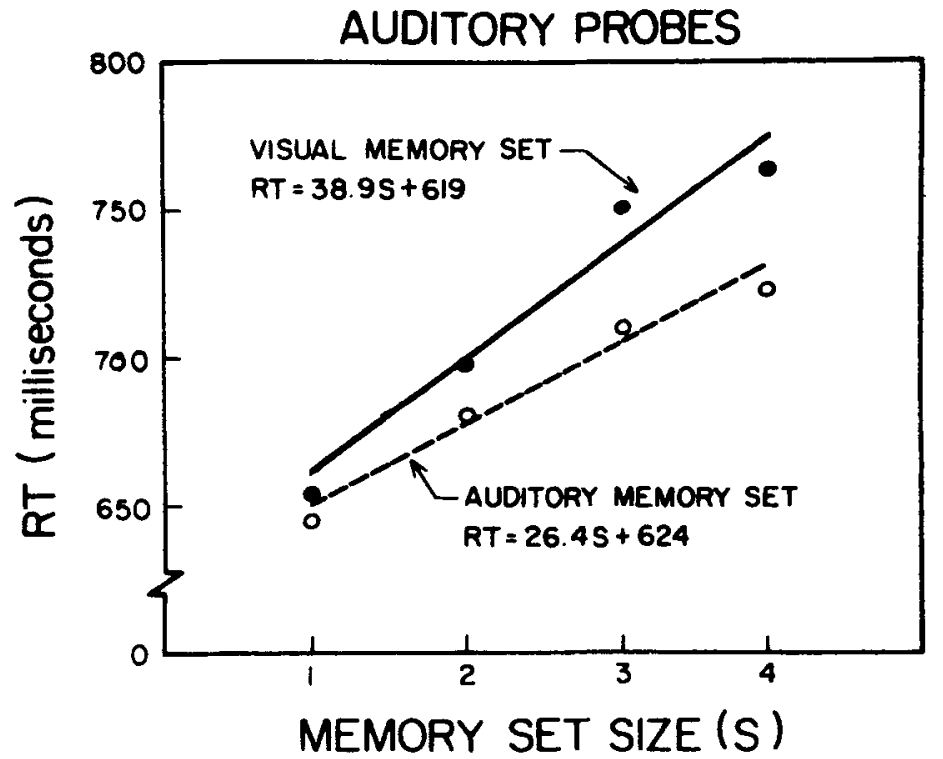

Fig. 1. Mean RT as a function of set size when probe item is presented auditorily.

The slope difference shown in Fig. 1 does fit the bimodal hypothesis that STM consists of modality-specific itores. The within-modality AA comparisons are faster than the across-modality VA comparisons. The lack of any slope interaction for the visual probe cases does not support the two-store hypothesis. The VV within-mode comparisons are no faster than the AV across mode comparisons and are slower than the AA comparisons.

Further negative evidence for the two-store hypothesis comes from the mixed-modality conditions. Retrieval should be severely disrupted with a mixed memory set since a great deal of

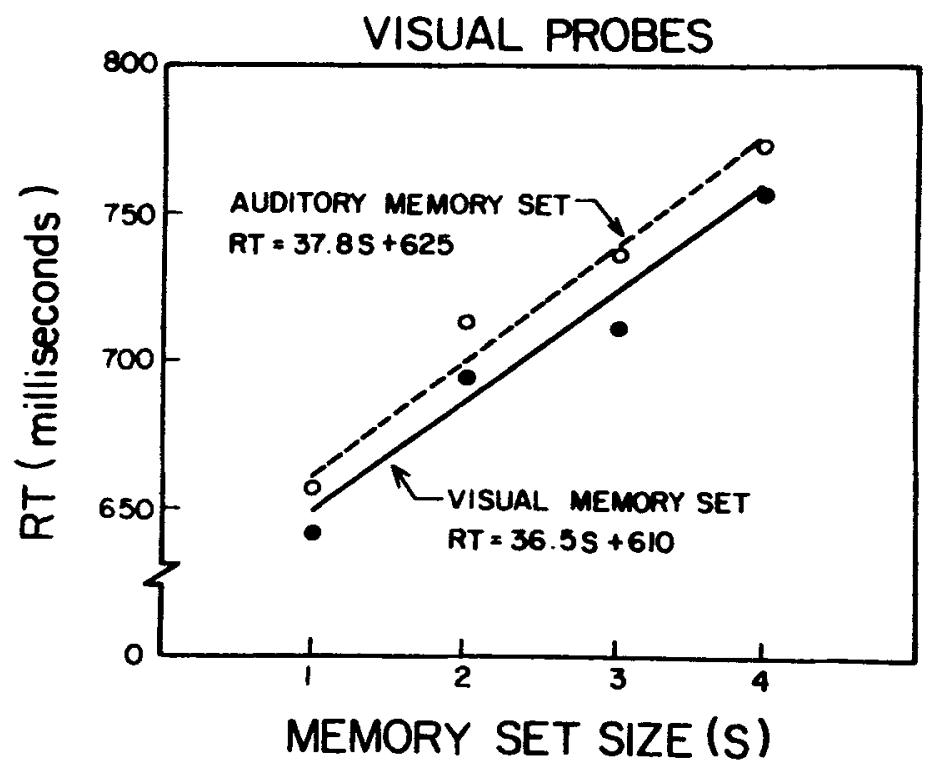

Fig. 2. Mean RT as a function of set size when probe is presented visually. switching from one modality to the other would be required. $\overline{\mathrm{RT}}$ s should be longer than those for either of the "pure" modality conditions. The data, however, show that retrieval rates in the mixed conditions are no slower than in the VV condition.

The results can be explained by the logogen model of Crowder and Morton (1969). When all memory items are presented auditorily, the information is converted into postcategorical form, and at the same time the sensory traces of the information persist in the auditory sensory store. If an auditory probe is presented, retrieval could be from this auditory store. This retrieval would be relatively rapid, since it could be done by physical matching of the auditory memory straces with the test item (Posner, 1969). If the probe is visual, retrieval from the auditory sensory store is not possible, since the memory items and the probe are not in a compatible format. Retrieval must be from the postcategorical store. Comparisons of probes to items in the categorized store is done at a slower rate than with items in the physical store, as reflected in the steeper slope for visual probes shown in Fig. 1. With visual presentation, the information is recoded into postcategorical form. The visual traces fade too quickly for there to be a long persisting visual sensory store. Thus, all retrieval must be from the postcategorical store.

When the presentation of the memory items is mixed, the auditory items will be represented by both a sensory trace and a postcategorical trace, while the visual items will be represented postcategorically only. The most efficient retrieval strategy might be to scan through the 


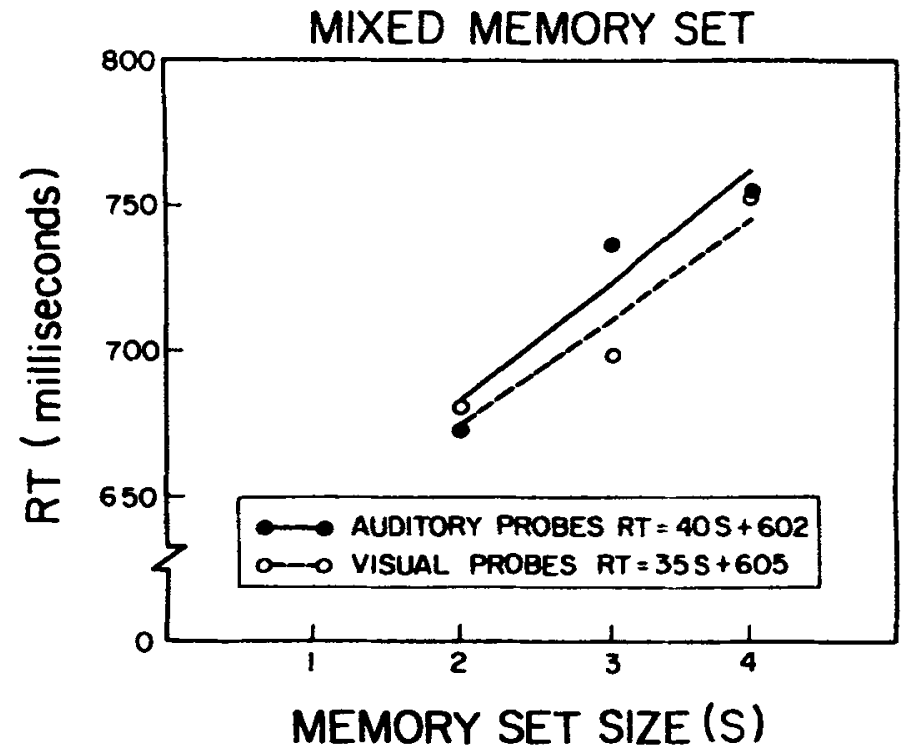

Fig. 3. Mean RT as a function of set size when modality of memory items is mixed.

postcategorical store. If this strategy is adopted, the mixed memory set condition becomes similar to the VV, $V A$, and AV conditions in that all lead to retrieval from a postcategorical store. This assertion seems to be confirmed by a comparison of Figs. 1 , 2 , and 3 , which show one retrieval rate for the AA condition and a slower retrieval rate that seems to be uniform for all the other conditions.

\section{EXPERIMENT 2}

The data from Experiment 1 seem incompatible with the hypothesis that all information in short-term memory is held in sensory-specific stores. It is possible that information could be held in sensory-specific stores if it were to S's advantage to do so, and that the task in Experiment 1 simply does not encourage such a strategy. In the mixed memory set condition of Experiment 1 it could be a disadvantage to store auditory and visual information separately, since this might require searching for matches in two different places when the probe is presented. By categorizing all items and storing them with a common format, $\mathrm{S}$ makes the scanning task run more smoothly. When all memory set information is presented in a single modality, $S$ might still convert all information into postcategorical form, since this would make the search task easier if the probe is not presented in the same modality as the memory items. This argument suggests that the format of storage might be governed by "control" processes that can be modified at the will of $S$ rather than by permanent, "structural" features of the memory system (Atkinson \&
Shiffrin, 1968). Experiment 2 was an attempt to present $S$ with a retrieval task in which holding information in separate auditory and visual sensory stores would be an advantage. On each trial, some memory items were presented auditorily and some visually. $A$ probe was then presented, and $S$ made a yes or no response. If the probe was in the set, it was always presented in the same modality as it was in the memory set. Logically it was necessary to match the probe against only those memory items which were presented in the same modality. If $\mathbf{S}$ can keep the auditory and visual memory items separated, he should be able to reduce the effective size of the search set. For any given number of items presented in the "relevant" modality, $\overline{R T}$ should not be affected by the number of items presented in the "irrelevant" modality. For any given memory set size, $\overline{\mathrm{RT}}$ should be inversely correlated with the number of items presented in the "irrelevant" modality.

\section{Method}

Subjects. The Ss were six students at the University of Toronto, both male and female. Each had had some previous experience in RT tasks. Each $\mathrm{S}$ was run for one practice session and six experimental sessions and was paid $\$ 2.00$ per session.

Apparatus. The apparatus was the same as that used in Experiment 1.

Procedure. Each trial began with a row of asterisks and the word "start" presented simultaneously. The memory set of 1 to 4 words followed at a rate of 3 words $/ 2$ sec. Then a row of " +8 " was shown and "probe" was spoken simultaneously. Finally, the probe word was presented, and $S$ indicated whether the probe word was in the memory set. Words instead of digits were used as stimulus items in order to establish the generality of the scan model and to eliminate the need to repeat the same stimuli trial after trial.

The words used were selected without replacement from a pool of 1,080 common two-syllable English words from which proper names and homonyms had been deleted. On each trial one modality was "relevant" and the other was "irrelevant." The probe was always presented in the "relevant" modality. The Ss were made fully aware that if the probe was in the memory set at all, it would be presented in the "relevant" modality and were encouraged to use this fact to employ an efficient retrieval strategy. The number of items presented in the "relevant" modality was $1,2,3$, or 4 . The number of "irrelevant" modality items was varied so that all possible combinations of relevant and irrelevant items adding up to a total set of $1,2,3$, or 4 resulted. Thus, if the number of "relevant" items was 1 , the number of irrelevant items could be $0,1,2$, or 3 , yielding total sets of $1,2,3$, or 4 . If the number of relevant items was 2 , the number of irrelevant items could be 0 , 1 , or 2 , yielding sets of 2,3 , or 4 . For each of the two relevant modalities, 10 types of memory sets resulted. The 10 combinations by 2 relevant modalities by 2 test types (positive or negative) yields 40 trials per replication. Each $S$ was tested on six replications per day. The order in which relevant and irrelevant items were presented was randomized for each trial. For each set size, each serial position was tested with equal frequency.

The error rates for the 40 conditions ranged from $1 \%$ to $6 \%$, with an average of about $4 \%$. Trials on which errors were made were discarded from the RT analysis. Figure 4 shows $\overline{\mathbf{R T}}$ as a function of total set size for the auditory relevant cases. Separate curves are shown for the condition where all items are relevant (pure auditory) and the conditions where not all items are relevant (mixed). Data for positive and negative trials have been combined. Linear regression accounted for $90.8 \%$ of the variance. The mixed curve was not lower than the pure curve, as would be the case if $S s$ were able to reduce the "effective" set size in the mixed conditions. In fact, the mixed curve was considerably higher than the pure curve. The difference in the intercepts of the two functions was 
significant, $t(3)=6.64, p<.01$. The difference in slopes did not reach significance, $t(3)=1.97, .10>p>$ .05 .

Figure 5 shows the "pure" and mixed curves for the visual relevant cases. Linear regression accounted for $94.8 \%$ of the variance. The two functions appear to be equivalent. Table 1 compares the $\overline{\mathrm{RT}}$ data for auditory and visual "relevant" sets for each condition where the total set size was four items. Data for positive and negative trials have been combined. Although there is some suggestion that $\overline{R T}$ decreases as the number of irrelevant items increases from one to three, the effect is small if not nonexistent and there is clearly no superiority of the "mixed" over the "pure" conditions.

\section{Discussion}

There is little indication that Ss can hold auditory and visual information in separate stores and restrict retrieval to a search of one store or the other, even when the experimental task heavily favors such a strategy. $\overline{\mathrm{RT}}$ is not reduced under conditions where information presented in one modality or the other can be ignored. If anything, the presence of such irrelevant information tends to increase $\overline{\mathrm{RT}}$. The results favor the same explanation offered for the results of Experiment 1. When all items are presented auditorily and the probe is presented auditorily, retrieval can be from a precategorical auditory store. When the memory items are presented visually or when visual and auditory items are mixed, retrieval is made from a postcategorical store. There are no differences between the

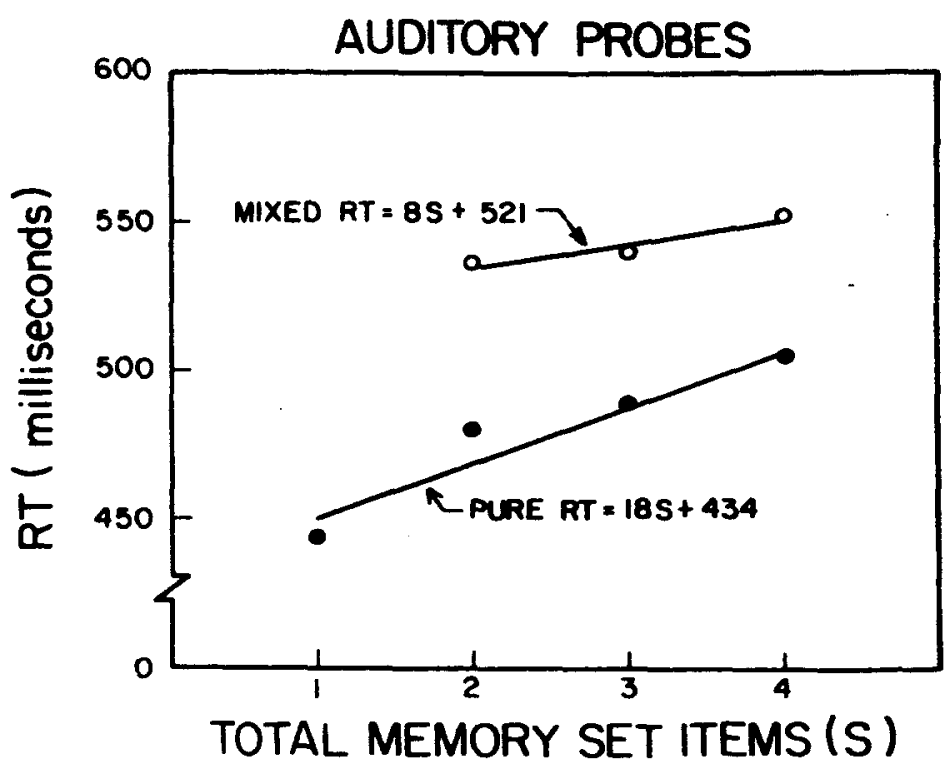

Fig. 4. Mean RT as a function of set size when auditory items are relevant. mixed and pure visual conditions, since retrieval is from the postcategorical store in both cases. $\overline{R T}$ in "the pure auditory list condition is less than $\overline{R T}$ for the mixed list conditions because of the advantages of retrieval from a sensory store. These differences in retrieval of auditorily and visually presented information seem to reflect structural aspects of the me mory system

Several differences in the results of Experiments 1 and 2 should be noted: The superiority of the pure auditory conditions is realized as an apparently faster scanning rate (slope effect) in Experiment 1 but as a smaller encoding time (intercept effect) in

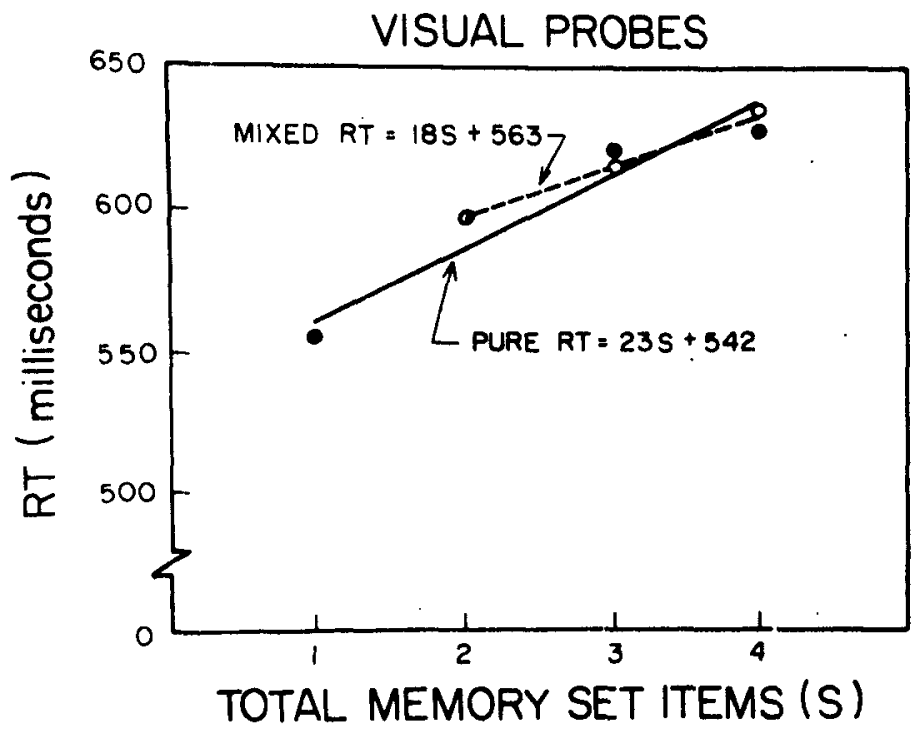

Fig. 5. Mean RT as a function of set size when visual items are relevant.
Experiment 2. One possible reason for this is that the conditions of Experiment 2 somehow resulted in more thorough encoding of the probe before scanning. The modality differences are thus reflected in the encoding times, and the representations yielded by the encoding process are uniformly sharp, resulting in relatively fast scanning rates in all cases. There are several procedural differences in the two experiments; the use of words vs digits is perhaps crucial in causing the change in pattern of results. It is remarkable that the ordering of difficulty is the same in the two experiments despite these other differences: pure auditory is always the fastest condition and the remaining conditions do not differ from one another.

\section{EXPERIMENT 3}

So far, it has been asserted that auditorily presented information persists in sensory form for a "few" seconds, while visually presented

Table 1

Mean Reaction Times to Auditory and Virual Test Items as a Function of Number of Items Presented in the Irrelevant Mode (Set Size $=4$ )

\begin{tabular}{ccc}
$\begin{array}{c}\text { Modality } \\
\text { of Test } \\
\text { Item }\end{array}$ & $\begin{array}{c}\text { Number of } \\
\text { Items in } \\
\text { Irrelevant } \\
\text { Modality }\end{array}$ & $\begin{array}{c}\text { Mean } \\
\text { RT } \\
\text { (Msec) }\end{array}$ \\
\hline Auditory & 0 & $\mathbf{5 0 6}$ \\
Auditory & 1 & 557 \\
Auditory & 2 & 564 \\
Auditory & 3 & 539 \\
Visual & 0 & 628 \\
Visual & 1 & 654 \\
Visual & 2 & 630 \\
Visual & 3 & 624 \\
\hline
\end{tabular}




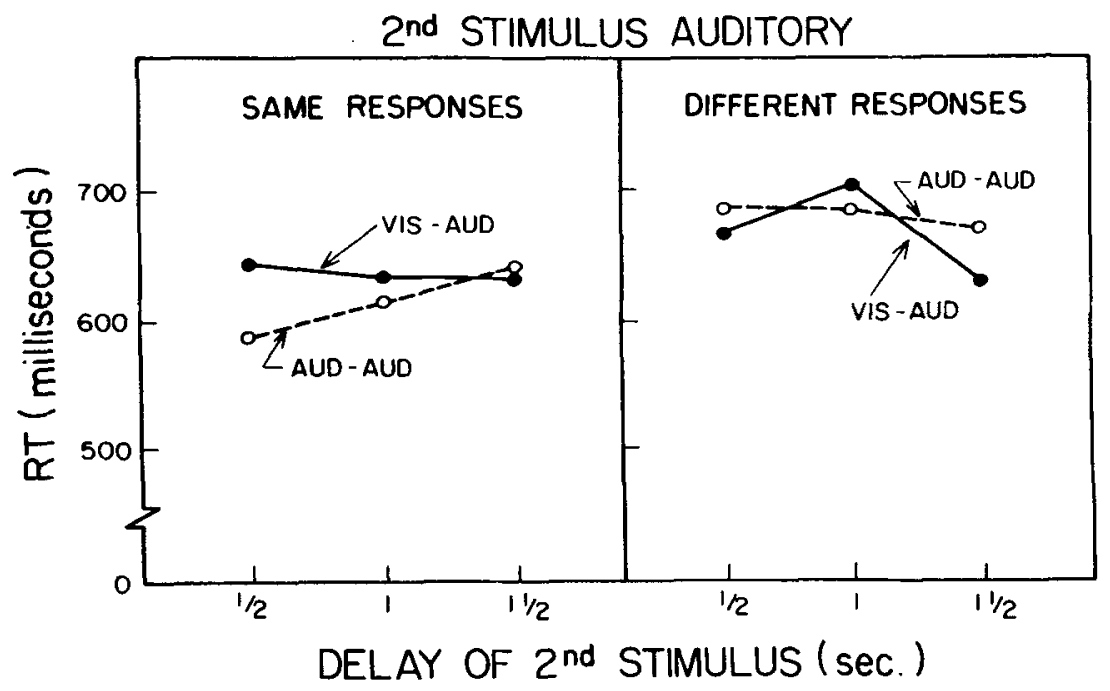

Fig. 6. Mean RT as a function of delay of second stimulus when second stimulus is auditory.

information persists in sensory form for less than 1 sec. Experiment 3 offered a test of this assertion by measuring these persistence times using Posner's (1969) delayed matching technique. $S$ is presented with two letters separated by some small time interval. He must decide as quickly as possible whether they are the same letter or different letters. Either letter can be presented auditorily or visually. If the trace of a letter can be matched more rapidly with a letter presented in the same modality than it can with a letter which has the same name but is presented in a different modality, then that trace must preserve some of the sensory attributes of the letter. By varying the interval between the two letters, one can observe whether or not this sensory information is lost over time and get an estimate of its persistence.

\section{Method}

Subjects. The Ss were eight students at the University of Toronto, both male and female. Each $S$ served in one session and was paid $\$ 2.00$.

Apparatus. The apparatus was the same as that used in Experiments 1 and 2.

Procedure. On each trial, S was presented with two letters in sequential fashion and was required to indicate whether they were the same letter or different letters. After a 2-sec interval, the next trial was presented. All stimuli used were selected from the population $A, I, U, H, P, R$. The first letter could be presented auditorily or visually, and the second letter could also be presented in either mode. The two stimuli were "same" or "different" equally often. The delay between presentation of the first and
The error rates were never greater than $6 \%$ for any trial type, and for single error made. All RT analyses were based on correct responses only. Figure 6 shows mean RTs as a function of delay when the second letter is presented auditorily. $\overline{\mathrm{RT} s}$ for "same" and "different" responses are shown on separate graphs. The labels on the graphs indicate the modality of the first and second stimuli, respectively. For the "same" responses, the AA trials result in faster $\overline{R T}$ s than VA when the delay interval is $1 / 2$ sec. This superiority is lost quite rapidly, disappearing between 1 and $11 / 2$ sec. The decline in AA superiority results mostly from rises in the $A A$ curve rather than from drops in the VA curve, which is fairly stable over delay intervals. The times for "different" responses are considerably slower than those for "same" responses. Here the AA curve is relatively flat, and there do not seem to be any systematic differences between the $A A$ and VA curves, although it is difficult to be sure because the VA curve is noisy.

Figure 7 shows comparable functions for trials where the second stimulus is presented visually. Again, the "different" responses are considerably slower than the "same" responses. There is little evidence for VV-AV differences. None of the curves varies with second stimulus delay.

$A$ repeated-measures analysis of variance was performed on the data for correct responses. The data were before performing the analysis in order to meet the normality assumption of the analysis of variance model. Figures 6 and 7 imply the existence of a four-way interaction: modality of the first stimulus interacts with the interval between the two stimuli only when the second stimulus is auditory and the response is "same." This is the effect shown on the left-hand panel of Fig. 6. The four-way interaction of First Stimulus by Second Stimulus by Response Type by Interval was collapsed across the replications factor several trial types there was not a

second letter was $.5,1$, or 1.5 sec. 2 by 2 by 2 by $3=24$ different types of trials. The trials were presented in modality of the first stimulus a fixed so that four different types of presented per block.

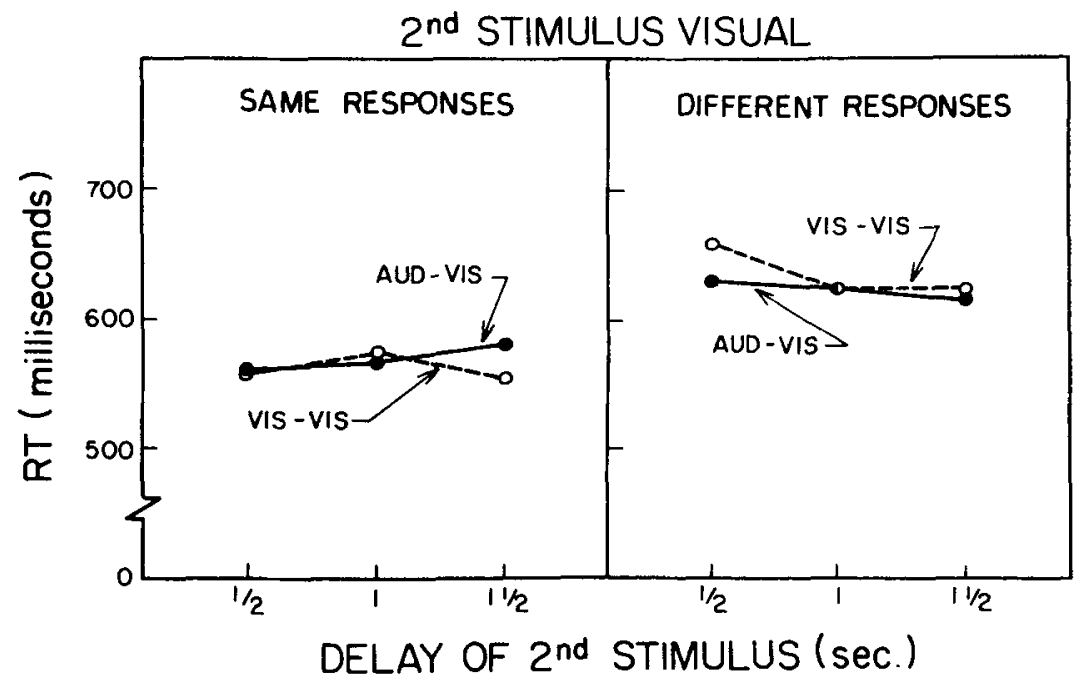

Fig. 7. Mean RT as a function of delay of second stimulus when second stimulus is visual. 
significant, $F(2,14)=5.3, p<.05$. Four of the six two-way interactions involving these four factors and two of the four three-way interactions were significant. In addition, the main effect of second stimulus was significant, $F(1,7)=126.2, p<.01$ indicating generally faster responses to visual stimuli. Finally, the observed effect that "same" responses were faster than "different" responses was also significant, $F(1,7)=52.2, p<.01$.

\section{Discussion}

The data support the hypothesis that retrieval by an auditory sensory match is possible for a short time after presentation of an auditory memory item An auditory probe can be matched more quickly with an auditory memory item than with a visual memory item. The fact that this AA retrieval process is slower as interstimulus delay lengthens suggests that the sensory information from the me mory item is being lost and $S$ must rely increasingly on a slower match of postcategorical attributes as time elapses. The VA trials always require postcategorical retrieval. Since this type of match does not depend on the existence of fading sensory information, it is not affected by delay interval.

The "different" AA trials are not affected by delay interval either. This implies that a postcategorical comparison is always made before a "different" response is initiated. This seems like a reasonable strategy if the auditory trace is rapidly fading. If a "physical" match is made, a positive response is initiated. If a physical mismatch is made, it is not certain whether the mismatch means that the two stimuli are in fact different or that the two stimuli are really the same and the physical trace is too degraded to be able to make a match-hence the necessity of attempting a postcategorical match before executing a "different" response.

The data for visual second stimuli show no evidence for a visual sensory store similar to the inferred auditory store. Pure visual trials do not result in faster $\overline{R T S}$ than AV trials. There are no changes with delay of second stimulus.

Experiment 3 supports the earlier hypothesis that there is precategorical auditory storage which lasts for a brief time and from which retrieval is possible. The corresponding visual storage is too brief to allow retrieval. Under conditions where retrieval from precategorical storage is not possible, the modality of the memory information does not affect retrieval.

\section{SUMMARY}

The experiments reported support a logogen account of the format of STM information structure. Auditorily presented information can exist in an auditory format which preserves sensory features for a short time or in a postcategorical format which does not preserve sensory features but presumably extracts some sort of semantic features. Visual information does not have a stable sensory representation and exists primarily in postcategorical form. The three experiments all support this interpretation using a variety of materials and settings.

The lack of evidence for visual sensory memory conflicts with evidence from earlier RT experiments: Posner and his associates have found faster matching between a test item and a visually presented memory item when the two are physically rather than simply nominally identical, suggesting that the memory item retains sensory features (Posner, 1969). In addition, Experiment I of Chase and Calfee (1969) shows superiority of pure visual (VV) over mixed auditory-visual (AV) matching in a situation similar to the present Experiment 1 . Whether these conflicts are due to simple methodological variations or theoretically interesting differences is unclear. The results of Experiment II of Chase and Calfee (1969) do not show any differences between the $A V$ and $V V$ conditions and are similar to the present results in that respect. This suggests that the lack of a difference between $V V$ and AV may be typical when the Sternberg procedure is used. The Posner findings, with which Experiment 3 seems to conflict, are based on visual presentation conditions almost exclusively. The presence of auditory stimuli in the present experiments may have caused a change in processing strategy; dividing attention between "looking for" and "listening for" stimuli may cause a decrement in short-term visual storage. Also, the fact that physical matching is a priori impossible on up to one-half of the trials in the present experiments may cause $S$ to place more stress on the semantic or "higher-order" aspects of the stimuli, with a resulting decrement in visual memory. The answers to these conjectures await further research.

One other result relevant to the present experiments has been reported by Kirsner and Craik (1971), who varied the modality of both memory set and probe item and required $\mathrm{Ss}$ to make a Sternberg-like yes-no decision in one part of the experiment and to name the probe item itself in another part. Kirsner and Craik found that for both tasks, the AA condition resulted in shorter RTs than the AV, VA, and VV conditions, which all resulted in similar $\overline{\mathrm{R} T \mathrm{~s}}$. They also obtained corrected retrieval RTs by subtracting the naming $\mathrm{RT}$ from the decision RTs and again found that $A V, V A$, and $V V$ conditions did not differ, while the AA condition was superior to all three. The Kirsner and Craik results therefore seem consonant with the present results and interpretation.

Finally, the fact that the RT-comparison procedure is sensitive to format features suggests its usefulness for studying other aspects of information format such as some of the features of postcategorical storage.

\section{REFERENCES}

ATKINSON, R. C., \& SHIFFRIN, R. M Human memory: A proposed system and its control processes. In $K$. W. Spence and J. T. Spence (Eds.), The psychology of learning and motivation: Advances in research and theory. Vol. 2. New Yodk: Academic Press, 1968

BADDELEY, A. D. Short-term memory for word sequences as a function of acoustic. semantic and formal similarity. Quarterly Journal of Experimental Psychology, $1966,18,362-365$.

CHASE, W. G., \& CALFEE, R. C. Modality and similarity effects in short-term recognition memory. Journal of Experimental Psychology, 1969, 81, 510-514.

CONRAD, R. Acoustic confusions in immediate memory. British Journal of Psychology, 1964, 55, 75-84.

CROWDER, R. G., \& MORTON, J Precategorical acoustic storage (PAS). Perception \& Psychophysics, 1969, 5, 365-373.

DRAPER, N. R., \& SMTTH, H. Applied regression analysis. New York: Wiley, 1966 .

KIRSNER, K., \& CRAIK, F. I, M. Naming and decision processes in short-term recognition memory. Journal of Experimental Psychology, 1971, 88, 149-157.

MURDOCK, B. B., JR, Visual and auditory stores in short-term memory. Quarterly Journal of Experimental Psychology, 1966, 18, 206-211.

MURDOCK, B. B., JR. Auditory and visual stores in short-term memory. Acta Psychologica, 1967, 27, 316-324.

MURDOCK, B. B., JR. Modality effects in short-term memory: Storage or retrieval? Joumal of Experimental Psychology, $1968,77,79-86$.

MURDOCK, B. B., JR. Where or when? Modality effects as a function of temporal and spatial distribution of information. Journal of Verbal Learning \& Verbal Behavior, 1969, 8, 378-383.

POSNER, M. I. Abstraction and the process of recognition. In $J$, T. Spence and $G$ Bower (Eds.), The psychology of learning and $m$ otivation: Advances in research and theory. Vol. 3. New York: Academic Press, 19.69.

SPERLING, $G$. The information available in brief visual presentations. Psychological Monographs, $1960,74(11$, Whole No. 498)

SPERLING, G. A model for visual memory tasks. Human Factors, 1963, 5, 19-31.

STERNBERG, S. High-speed scanning in human memory, Science, 1966, 153, 652-654.

STERNBERG, S. Two operations in character-recognition: Some evidence from reaction-time measurements. Perception \& Psychophysics, 1967, 2, 45-53.

STERNBERG, S. Memory-scanning: Mental processes revealed by reaction-time 
experiments. American Scientist, 1969, 57, 421-457.

NOTES

1. Sternberg's assertion was made only in the context of visual presentation; the possibility that memory items are stored visually regardless of presentation mode is raised only for purposes of completeness. It does not imply that Sternberg would agree with such a position.

2. Throughout the paper, the terms AA, $A V, V A$, and $V V$ will be used to refer to the various modality combinations. The first letter indicates the modality of the memory set, and the second latter indicates the modality of the probe.

(Accepted for publication January 17 